\title{
Las nuevas generaciones de mujeres, el embarazo no previsto, las edades y la segmentación social en la sociedad chilena
}

IRMA PALMA

\section{Unpredicted pregnancy among Chilean young women}

Background: Unpredicted pregnancy is an evolution of the concepts undesired and unplanned pregnancy. It is more common among vulnerable strata of our society and related to lack of education. Aim: To explore the prevalence and social concomitants of unpredicted pregnancy among young women. Material and Methods: Analysis of the databases of the Sixth National Youth Survey carried out by the Instituto Nacional de la Juventud in Chile during 2009. The universe corresponds to 7570 participants aged between 15 and 29 years, of both genders. Results: Unpredicted pregnancy occurred in $43 \%$ of sexually active surveyed women. It was more common among women with a lower educational level and those aged 15 to 24 years, especially during the onset of active sexual life. There was also an inverse relationship between the degree of education, the use of contraception and the age when sexual activity starts. Conclusions: Unpredicted pregnancy is frequent among teenagers and more common among less educated individuals. The frequency of use of contraception is associated in greater measure to the degree of education rather than the age of onset of sexual activity.

(Rev Med Chile 2012; 140: 319-325).

Key words: Contraception; Pregnancy in adolescence; Pregnancy, unplanned.



Departamento de Psicología, Universidad de Recibido el 9 de mayo de 2011, aceptado el 16 de noviembre de 2011

Correspondencia a: Irma Palma. E-mail: ipalma@ichile.cl
E l objeto de este trabajo es el embarazo no previsto (ENP) en las mujeres jóvenes en entre su existencia, la estructuración social de las edades en que se inscribe. Esto sólo es posible hacerlo directa e independientemente de condiciones de embarazo, aborto, paternidad o maternidad a partir de la V Encuesta Nacional de Juventud, en 2006, pues otras encuestas y sistemas de registro hasta ahora no lo hacían posible ${ }^{1-3}$. Esto permitirá iniciar una línea de investigación que contribuya analíticamente a su conocimiento y de esta forma aportar fundamento empírico a las políticas públicas.

Usamos el concepto de ENP ${ }^{4,5}$, dos conceptos le han precedido y que remiten a los campos de la psicología y la salud pública: embarazo no deseado y embarazo no planificado. Entre uno y otro, el
ENP. Antes que no planificación, no previsión. No previsto es tanto un juicio interno sobre la disponibilidad subjetiva para un embarazo conducente a la paternidad o la maternidad, como una gestión de una práctica preventiva; en el caso del ENP, el sujeto no se encuentra ni disponible ni prevenido.

Formulamos tres análisis destinados a conocer: a) la proporción de jóvenes que lo experimenta al menos una vez en la vida; b) en qué medida sucede en etapas tempranas de la vida, aunque no exprese su estructuración etaria permite una aproximación provisional (pues una cohorte joven no captura bien las edades de un evento que se produce también posteriormente) ${ }^{5}$; c) el momento en que en el curso de las trayectorias sexuales acontece; d) si la edad de la primera relación sexual se encuentra relacionada con su ocurrencia.

Luego analizamos la relación entre edades de 
la primera relación sexual y uso de tecnología preventiva, de modo de conocer si la precocidad se encuentra asociada a un menor uso. En tercer lugar, exploramos la relación entre la existencia del ENP en las nuevas generaciones, la estructuración social de las edades en que se inscribe y la estratificación social prevalente en la sociedad chilena. Nuestra aproximación pone en relación diversas trayectorias; el continuo de experiencias que van trazando itinerarios que se construyen simultánea y pluralmente en múltiples dimensiones ${ }^{6}$. Por una parte, trayectorias sexuales, reproductivas y conyugales, y por otra, educacionales. Esto permite conocer si es propiamente la edad un elemento intrínsecamente asociado a la exposición al ENP ${ }^{7}$ o si su ocurrencia se encuentra asociada a una estructuración socialmente diferenciada del conjunto de estas trayectorias.

\section{Material y Método}

Usamos la Base de Datos de la Sexta Encuesta Nacional de Juventud, Instituto Nacional de la Juventud, 2009. El universo son hombres y mujeres entre 15 y 29 años, del país. El muestreo es estratificado, por conglomerados y poli-etápico. Su muestra es 7.570 sujetos, y asegura una representatividad a nivel nacional y regional.

En este estudio sucede que su objeto y método coinciden en una misma temporalidad-el ENP en una edad y en una etapa de la vida-, y una encuesta que considera sólo a sujetos jóvene -están aún en proceso de producción de las prácticas conducentes a la ocurrencia del mismo (una proporción importante de éstos no ha tenido aún una primera relación sexual). Por ello, consideramos aquí sólo al segmento post-adolescente (20-29 años) pues se minimiza la dificultad, porque a partir de los 20 años se dispone de una muestra en que un número elevado de sujetos se encuentran sexualmente activos y por ello se dispone de una cohorte etaria que en un nivel importante ya ha tenido relaciones sexuales, ha usado o no tecnología y ha tenido oportunidad de enfrentar un ENP. Usamos la cohorte 25-29 años en algunos análisis, cuando buscamos disponer de una mayor distancia temporal para observar el evento en estudio.

Asimismo, usamos el nivel de escolaridad alcanzado por los sujetos y no la pertenencia a un grupo socioeconómico, pues tratamos de estable- cer una relación entre la existencia del ENP con las trayectorias educacionales - por su edad, las mujeres estudiadas tienen edades regulares de ingreso a la educación superior. La investigación social ha puesto de manifiesto que estas trayectorias se encuentran estratificadas en la sociedad chilena, y que en la actualidad se realizan en un contexto de expansión educacional y al mismo tiempo que de la existencia de barreras tanto para concluir la educación secundaria, como en el acceso a la educación superior, barreras que constituyen fuentes de desigualdad asociadas al origen social ${ }^{8,9}$.

Usa un cuestionario de aplicación cara a cara con predominio de preguntas de respuesta estandarizada. Incluye dos preguntas relacionadas con el ENP: ¿Te ha sucedido alguna vez que tú o tu pareja haya quedado embarazada sin planificarlo? y ¿Qué edad tenías tú cuando esto te sucedió o le sucedió a tu pareja? No indaga si hubo más de un evento en la vida. Nuestro supuesto es que en caso que haya existido más de un evento, se trataría del más antiguo, pues el primer evento es más significativo y crítico biográficamente, y sería lo que se recuerda cuando se es consultado. Incluye preguntas sobre si se ha tenido relaciones sexuales y edad, uso de tecnología preventiva (TP).

El procesamiento de la información hace uso de técnicas estadísticas descriptivas uni-variadas y bi-variadas. Para describir una variable se utilizan medidas de tendencia central. Para describir dos variables conjuntamente se utilizan Tablas de Contingencia y Comparación de Medias. La elección del procedimiento estadístico depende del nivel de medida de las variables utilizadas. Para el análisis se usa el software de procesamiento de datos SPSS 15.0.

\section{Resultados}

El ENP se presenta como una experiencia frecuente en la generación joven de mujeres en la sociedad chilena. Lo ha experimentado en la vida $42,5 \%$ de quienes tienen entre 15 y 29 años, que se encuentran sexualmente activas (Tabla 1).

A su vez, existe una alta diferenciación social en la ocurrencia del ENP. Las mujeres que alcanzan niveles educacionales superiores experimentan el ENP con menor frecuencia; en sentido inverso, las que alcanzan niveles de educación inferiores lo experimentan con mucha mayor frecuencia. 
En términos de su distribución poblacional, el ENP se ubica fundamentalmente en los niveles educacionales medios y básicos (Tabla 2).

El ENP es una experiencia reproductiva temprana: nueve de cada diez jóvenes lo experimentan entre los 15 y 24 años,

Tabla 1. Mujeres que experimentan al menos un embarazo no previsto en la vida, según edades. Cohorte 15 a 29 años

\begin{tabular}{|lrrrrr|}
\hline & \multicolumn{2}{c}{ Sí } & \multicolumn{2}{c|}{ No } & \\
& $\%$ fila & $\%$ col. & $\%$ fila & $\%$ col. & $\begin{array}{c}\text { n no } \\
\text { ponderado }\end{array}$ \\
\hline $15-17$ años & 20,9 & 4,9 & 79,1 & 13,7 & 363 \\
\hline $18-19$ años & 31,8 & 8,8 & 68,2 & 14,0 & 459 \\
\hline $20-24$ años & 42,1 & 38,6 & 57,9 & 39,2 & 1.171 \\
\hline $25-29$ años & 51,6 & 47,6 & 48,4 & 33,0 & 844 \\
\hline Total & 42,5 & 100,0 & 57,5 & 100,0 & 2.837 \\
\hline
\end{tabular}

Tabla 2. Nivel de embarazo no previsto en mujeres sexualmente activas, según nivel educacional. Cohorte 20-29 años

\begin{tabular}{|lcccrc|}
\hline & \multicolumn{2}{c}{ Sí } & \multicolumn{2}{c|}{ No } & \\
& $\%$ fila & $\%$ col. & $\%$ fila & $\%$ col. & $\begin{array}{c}\text { n no } \\
\text { ponderado }\end{array}$ \\
\hline B. y M. Inc. & 71,7 & 30,7 & 28,3 & 10,7 & 457 \\
\hline M. Comp. & 51,2 & 43,3 & 48,8 & 36,4 & 756 \\
\hline Superior & 30,3 & 26,0 & 69,7 & 52,9 & 795 \\
\hline Total & 46,9 & 100,0 & 53,1 & 100,0 & 2.008 \\
\hline
\end{tabular}

la mitad sucede en la adolescencia y uno entre cuatro a la edad de 17 años o menos. A su vez, es más temprana cuando sólo alcanzan niveles de educación básica o no completan la enseñanza media: la mitad sucede a los 17 años o menos, uno entre cinco en quienes alcanzan la educación media, y sólo uno entre siete en quienes tienen educación superior (Tabla 3 ).

El ENP sucede en el inicio e instalación de las trayectorias sexuales antes de los dos años- en la mitad de las jóvenes, y es especialmente próximo entre las que alcanzan menores niveles educacionales, que son justamente quienes usan menos frecuentemente TP (Tabla 4).

La estructuración de las edades de la primera relación sexual no es homogénea en las jóvenes. La primera relación sexual es más temprana en la vida cuanto más bajo sea el nivel educacional. A su vez, las edades de quienes experimentan un ENP y quienes no lo hacen son relativamente próximas entre las que alcanzan niveles inferiores de educación, pues en general todas lo hacen más tempranamente en la vida; en cambio, cuando alcanzan la educación superior quienes no experimentan un ENP lo hacen más tardíamente que las que sí se embarazan (Figura 1).

Cuanto más temprano sea el comienzo de la trayectoria sexual, menor es el uso de TP. Sin embargo, esta re-

Tabla 3. Edades de ocurrencia del embarazo no previsto en mujeres, según edades y nivel educacional. Cohorte 25-29 años.*

\begin{tabular}{|c|c|c|c|c|c|c|c|c|}
\hline & \multicolumn{2}{|c|}{ B. y M. Inc. } & \multicolumn{2}{|c|}{ M. Comp. } & \multicolumn{2}{|c|}{ Superior } & \multicolumn{2}{|c|}{ Total } \\
\hline & $\%$ col. & $\begin{array}{l}\text { n no } \\
\text { pon. }\end{array}$ & \% col. & $\begin{array}{l}\text { n no } \\
\text { pon. }\end{array}$ & $\%$ col. & $\begin{array}{l}\text { n no } \\
\text { pon. }\end{array}$ & $\%$ col. & $\begin{array}{l}\text { n no } \\
\text { pon. }\end{array}$ \\
\hline 17 o menos & 46,5 & 68 & 19,4 & 38 & 14,4 & 20 & 26,2 & 126 \\
\hline 18 a 19 & 24,7 & 34 & 23,4 & 41 & 22,9 & 21 & 23,7 & 96 \\
\hline 20 a 24 & 21,4 & 38 & 41,8 & 76 & 51,8 & 54 & 38,2 & 168 \\
\hline 25 a 29 & 7,4 & 9 & 15,4 & 26 & 10,9 & 15 & 11,9 & 50 \\
\hline Total & 100,0 & 149 & 100,0 & 181 & 100,0 & 110 & 100,0 & 440 \\
\hline
\end{tabular}

*Usamos la cohorte 25 a 29 años, pues permite cubrir un período más largo de la población estudiada. 
Tabla 4. Desfase entre edad de la primera relación sexual y edad de embarazo no previsto en mujeres, según nivel educacional. Cohorte 20 - 29 años

\begin{tabular}{|lccccccccc|}
\hline Desfase & \multicolumn{2}{c}{ B. $\mathbf{y ~ M . ~ i n c . ~}$} & \multicolumn{2}{c}{ M. comp. } & \multicolumn{2}{c}{ Superior } & \multicolumn{2}{c|}{ Total } \\
& $\%$ col. & $\begin{array}{l}\text { n no } \\
\text { pon. }\end{array}$ & $\%$ col. & $\begin{array}{l}\text { n no } \\
\text { pon. }\end{array}$ & \% col. & $\begin{array}{l}\text { n no } \\
\text { pon. }\end{array}$ & \% col. & $\begin{array}{l}\text { n no } \\
\text { pon. }\end{array}$ \\
\hline Menos de 2 años & 61,0 & 179 & 52,5 & 186 & 44,1 & 112 & 52,8 & 477 \\
\hline 2 o más años & 39,0 & 121 & 47,5 & 191 & 55,9 & 132 & 47,2 & 444 \\
Total & 100,0 & 300 & 100,0 & 377 & 100,0 & 244 & 100,0 & 921 \\
\hline
\end{tabular}

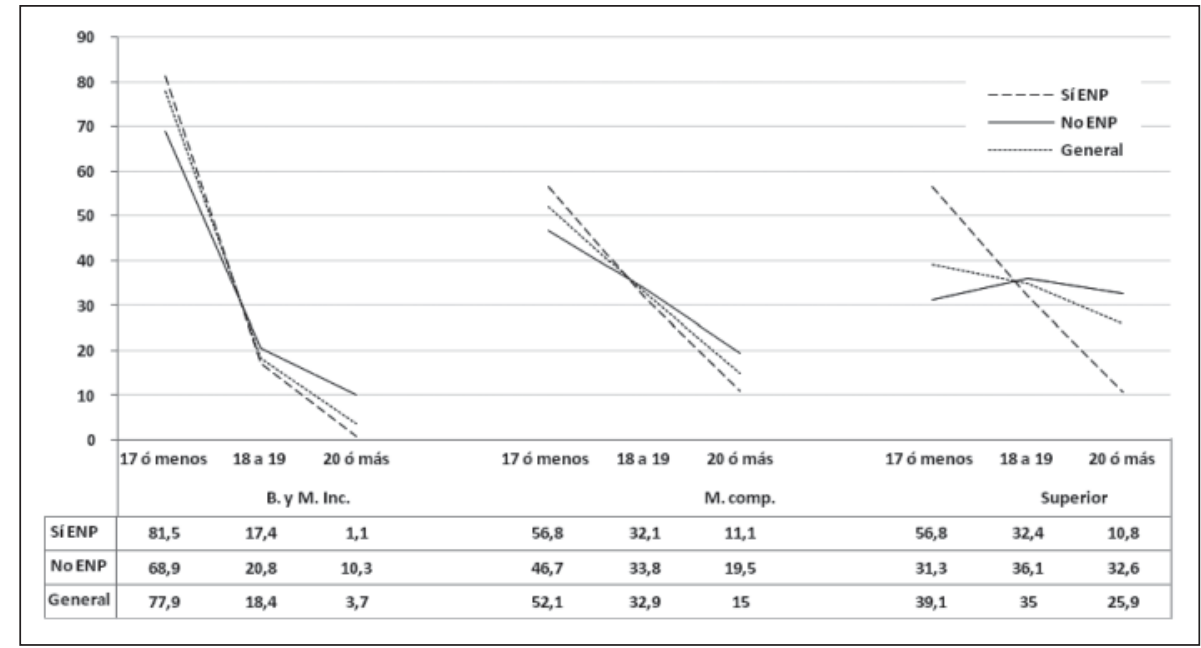

Figura 1. Edades de primera relación sexual en mujeres, según ocurrencia de embarazo no previsto y nivel educacional. 20 - 29 años (\% col.) ( $\mathrm{N}$ no ponderado: 1926). lación se encuentra fuertemente mediada por el nivel educacional alcanzado. Cuando cualesquiera de ellas tienen la primera relación sexual a los 17 años o antes, sólo una de cada cuatro de quienes no concluyen la enseñanza media usa TP, en tanto aumenta a una de cada dos en quienes alcanzan niveles de educación superior, mucho más incluso que aquellas con niveles educacionales inferiores que lo hacen en edades más tardías, que sólo la usan dos de cada cinco (Tabla 5).

En sus trayectorias biográficas las jóvenes presentan una importante diferenciación social de las edades de la fertilidad en el curso de sus trayectorias reproductivas. Las mujeres con niveles de educación superior presentan una fecundidad más tardía, escasamente iniciada en la adolescencia, y altamente postergada hasta pasado el período de juventud. En sentido inverso, aquellas con niveles de educación básica o media incompleta presentan una fecundidad más temprana, en una alta proporción en la adolescencia, que no se extiende por todo el período, sino que tiende a concluir antes de los 25 años. Entre unas y otras se encuentran las que cursan la enseñanza media completa, que presentan una fecundidad no muy temprana ni muy tardía, menos en la adolescencia que las últimas y menos en la etapa tardía como en las primeras (Tabla 6).

Del mismo modo, sus trayectorias conyugales (que entendemos genéricamente como uniones, sean maritales o cohabitación) presentan una importante diferenciación social. Aquellas con niveles de educación superior presentan más bajos niveles y más tardías las edades. En sentido inverso, aquellas con niveles de educación básica o media incompleta presentan más altos niveles y más tempranas las edades de uniones. Entre unas y otras se encuentran las que cursan la enseñanza media completa, sin embargo, más próximas a las últimas (Tabla 7). 
ARTÍCULOS DE INVESTIGACIÓN

Embarazo no previsto en jóvenes chilenas - I. Palma et al

Tabla 5. Nivel de uso de tecnología preventiva en primera relación sexual en mujeres, según edad de primera relación sexual y nivel educacional. 20 - 29 años

\begin{tabular}{|c|c|c|c|c|c|c|c|}
\hline \multirow[t]{2}{*}{ N. Ed. } & \multirow[t]{2}{*}{ Edad $1^{a}$ RS } & \multicolumn{3}{|c|}{ Usa TP } & \multicolumn{3}{|c|}{ No usa TP } \\
\hline & & $\%$ fila & $\%$ col. & n no pon. & $\%$ fila & \% col. & n no pon. \\
\hline \multirow[t]{4}{*}{ B. y M. inc. } & 17 o menos & 24,9 & 73,1 & 86 & 75,1 & 80,0 & 252 \\
\hline & 18 a 19 & 31,7 & 21,7 & 30 & 68,3 & 16,9 & 51 \\
\hline & 20 o más & 38,1 & 5,2 & 4 & 61,9 & 3,1 & 19 \\
\hline & Total & 26,6 & 100,0 & 120 & 73,4 & 100,0 & 322 \\
\hline \multirow[t]{4}{*}{ M. comp. } & 17 o menos & 33,8 & 41,9 & 144 & 66,2 & 60,2 & 256 \\
\hline & 18 a 19 & 52,4 & 40,6 & 122 & 47,6 & 27,1 & 109 \\
\hline & 20 o más & 50,3 & 17,5 & 56 & 49,7 & 12,7 & 48 \\
\hline & Total & 42,4 & 100,0 & 322 & 57,6 & 100,0 & 413 \\
\hline \multirow[t]{4}{*}{ Superior } & 17 o menos & 55,0 & 33,8 & 161 & 45,0 & 48,0 & 157 \\
\hline & 18 a 19 & 67,0 & 36,9 & 193 & 33,0 & 31,5 & 95 \\
\hline & 20 ó más & 71,3 & 29,3 & 117 & 28,7 & 20,5 & 46 \\
\hline & Total & 63,5 & 100,0 & 471 & 36,5 & 100,0 & 298 \\
\hline \multirow[t]{4}{*}{ Total } & 17 o menos & 37,6 & 41,0 & 391 & 62,4 & 62,3 & 665 \\
\hline & 18 a 19 & 56,7 & 36,5 & 345 & 43,3 & 25,5 & 255 \\
\hline & 20 o más & 62,8 & 22,5 & 177 & 37,2 & 12,2 & 113 \\
\hline & Total & 47,8 & 100,0 & 913 & 52,2 & 100,0 & 1.033 \\
\hline
\end{tabular}

Tabla 6. Maternidad, según edades y niveles educacionales. Cohorte 25 a 29 años

\begin{tabular}{|c|c|c|c|c|c|c|c|c|c|c|c|c|c|c|c|}
\hline & \multicolumn{3}{|c|}{19 o menos } & \multicolumn{3}{|c|}{20 a 24 más } & \multicolumn{3}{|c|}{25 o más } & \multicolumn{3}{|c|}{ Sin hijos } & \multicolumn{3}{|c|}{ Total } \\
\hline & $\begin{array}{c}\% \\
\text { fila }\end{array}$ & $\begin{array}{c}\% \\
\text { col. }\end{array}$ & $\begin{array}{c}\text { n no } \\
\text { pond. }\end{array}$ & $\begin{array}{c}\% \\
\text { fila }\end{array}$ & $\begin{array}{c}\% \\
\text { col. }\end{array}$ & $\begin{array}{c}\text { n no } \\
\text { pond. }\end{array}$ & $\begin{array}{c}\% \\
\text { fila }\end{array}$ & $\begin{array}{c}\% \\
\text { col. }\end{array}$ & $\begin{array}{c}\text { n no } \\
\text { pond. }\end{array}$ & $\begin{array}{c}\% \\
\text { fila }\end{array}$ & $\begin{array}{c}\% \\
\text { col. }\end{array}$ & $\begin{array}{c}\text { n no } \\
\text { pond. }\end{array}$ & $\begin{array}{c}\% \\
\text { fila }\end{array}$ & $\begin{array}{c}\% \\
\text { col. }\end{array}$ & $\begin{array}{c}\text { n no } \\
\text { pond. }\end{array}$ \\
\hline B. y M. Inc. & 62,9 & 43,3 & 139 & 29,0 & 17,9 & 59 & 3,5 & 8,5 & 5 & 4,6 & 4,0 & 12 & 100,0 & 21,6 & 215 \\
\hline M. Comp. & 28,7 & 39,8 & 105 & 46,6 & 57,9 & 154 & 10,8 & 52,3 & 38 & 14,0 & 24,6 & 46 & 100,0 & 43,5 & 343 \\
\hline Superior & 15,2 & 16,9 & 50 & 24,3 & 24,2 & 69 & 10,1 & 39,2 & 33 & 50,4 & 71,3 & 135 & 100,0 & 34,9 & 287 \\
\hline Total & 31,4 & 100,0 & 294 & 35,0 & 100,0 & 282 & 9,0 & 100,0 & 76 & 24,7 & 100,0 & 193 & 100,0 & 100,0 & 845 \\
\hline
\end{tabular}

Tabla 7. Mujeres unidas alguna vez en la vida*, según cohortes de edad y nivel educacional. Cohorte 20 - 29 años

\begin{tabular}{|c|c|c|c|c|c|c|c|c|c|c|c|c|c|}
\hline & & & y $\mathbf{M}$. & & & n. Con & & & iuperi & & & Total & \\
\hline & & $\begin{array}{c}\% \\
\text { fila }\end{array}$ & $\begin{array}{c}\% \\
\text { col. }\end{array}$ & $\begin{array}{l}\text { n no } \\
\text { pon. }\end{array}$ & $\begin{array}{c}\% \\
\text { fila }\end{array}$ & $\begin{array}{c}\% \\
\text { col. }\end{array}$ & $\begin{array}{l}\text { n no } \\
\text { pon. }\end{array}$ & $\begin{array}{c}\% \\
\text { fila }\end{array}$ & $\begin{array}{c}\% \\
\text { col. }\end{array}$ & $\begin{array}{l}\text { n no } \\
\text { pon. }\end{array}$ & $\begin{array}{c}\% \\
\text { fila }\end{array}$ & $\begin{array}{c}\% \\
\text { col. }\end{array}$ & $\begin{array}{l}\text { n no } \\
\text { pon. }\end{array}$ \\
\hline 20 a 24 & No & 10,1 & 41,6 & 105 & 29,4 & 58,3 & 288 & 60,5 & 85,8 & 531 & 100,0 & 68,8 & 924 \\
\hline & Sí & 31,3 & 58,4 & 147 & 46,5 & 41,7 & 192 & 22,2 & 14,2 & 92 & 100,0 & 31,2 & 431 \\
\hline 25 a 29 & No & 10,4 & 21,9 & 44 & 37,4 & 38,7 & 141 & 52,1 & 63,8 & 190 & 100,0 & 44,3 & 375 \\
\hline & Sí & 29,5 & 78,1 & 179 & 47,0 & 61,3 & 221 & 23,5 & 36,2 & 124 & 100,0 & 55,7 & 524 \\
\hline Total & No & 10,2 & 31,2 & 149 & 32,4 & 48,0 & 429 & 57,4 & 76,9 & 721 & 100,0 & 57,2 & 1299 \\
\hline & Sí & 30,2 & 68,8 & 326 & 46,8 & 52,0 & 413 & 23,0 & 23,1 & 216 & 100,0 & 42,8 & 955 \\
\hline
\end{tabular}

${ }^{*}$ Casadas, solteras cohabitantes, separadas de hecho, viudas, divorciadas, anuladas. 


\section{Discusión}

El ENP se presenta como una experiencia frecuente en la generación joven de mujeres en la sociedad chilena. Al mismo tiempo, se encuentra socialmente diferenciado: muy poco frecuente en las que alcanzan niveles de educación superior, y especialmente frecuente entre las que sólo alcanzan niveles de educación básica o media incompleta. No constituye, pues, un evento transversal al conjunto de la sociedad. Es una experiencia reproductiva temprana en la vida, notablemente más temprana cuando tienen niveles de educación básica o media incompleta. Cuanto más temprano sea el comienzo de la trayectoria sexual, menor es el uso de TP y más frecuente es el ENP. Sin embargo, ¿es propiamente la edad un elemento intrínsecamente asociado a la prescindencia de TP y en consecuencia a la exposición al ENP?.

Una perspectiva comprende esta asociación entre una iniciación sexual más temprana y una mayor ocurrencia del ENP -mediada por un uso reducido de $\mathrm{TP}$ - como fruto de la inmadurez psicológica e inhabilidad del sujeto muy joven para la práctica preventiva (por ser menos experto y menos autónomo $)^{7}$. Sin embargo, así formulada la relación entre precocidad y ENP se encuentra sometida a discusión en el campo de la investigación social en sociedades europeas, en las que el uso de TP es elevado y extendido en el conjunto de las edades ${ }^{10}$, y en sociedades latinoamericanas esta asociación se presenta más mediada por la estratificación social ${ }^{11}$.

Una perspectiva alternativa sugeriría que la dificultad está en un acceso a la TP segmentado por edad, que impediría a un sujeto muy joven justamente el agenciamiento personal de su prevención, al mismo tiempo que habría un acceso segmentado socialmente. No es posible conocer aquí si existe un acceso etariamente y socialmente segmentado, pues la Encuesta Nacional de Juventud no estudia el acceso a TP. La primera perspectiva - por su esencialización de la edad del sexo, como una limitación intrínseca al sujeto-, no permite dar cuenta de la segmentación social en la existencia del ENP. La última resulta simplificadora, pues sitúa la dificultad enteramente fuera del sujeto, y nada indica que la provisión de TP pueda operar a distancia de las disposiciones subjetivas del individuo.

En la sociedad chilena esta relación entre edad y ENP se encuentra estrictamente asociada a la ubicación de las jóvenes en la estructura social. Como hemos mostrado, cuando quienes hacen su entrada en la sexualidad activa tempranamente son las de niveles de educación superior, lo experimentan menos frecuentemente que aquellas que no concluyen la enseñanza media que lo hacen en edades más tardías, y a su vez, cuando quienes hacen tempranamente su entrada en la sexualidad activa son las primeras, usan TP más frecuentemente que las últimas.

Comprender el lugar propiamente de la edad en la existencia del ENP no resulta analíticamente sencillo en el marco de una diferenciación social elevada del fenómeno. Una vez establecida, lo fundamental a comprender es cómo operaría esta diferenciación. Hemos mostrado precedentemente que existe una relación entre la exposición al ENP con las formas en que se configuran las trayectorias sexuales, preventivas, reproductivas y conyugales, en el marco de una estructuración socialmente diferenciada, sin embargo, en transformación, de las trayectorias educacionales femeninas en las nuevas generaciones.

Por otra parte, justamente en el contexto de la existencia de una diferenciación social de las trayectorias educacionales, proponemos observar las configuraciones de las trayectorias sexuales, preventivas, reproductivas y conyugales en las nuevas generaciones de mujeres a partir de una tensión entre orientaciones culturales, condiciones sociales y disposiciones subjetivas más individualizadas o más relacionales. Más precisamente, una perspectiva que puede ensayarse es vinculando tales configuraciones a procesos de individualización en curso, que tienen, sin embargo, en sociedades como la nuestra versiones más precarias ${ }^{12,13}$. Hay jóvenes cuyas vidas se encuentran altamente estructuradas en su subjetividad y organizadas en función de una proyectividad profesional; hay otras, en tanto, cuyas vidas se encuentran más estructuradas en su subjetividad y organizadas en función de una proyectividad relacional, familiar. A su vez, los márgenes de incidencia personal son percibidos por las primeras, como más amplios y el agenciamiento, más factible. Unas y otras harían una internalización de las trayectorias sociales/ educativas más probables en su medio ambiente social.

En los grupos de ingresos altos en su gran mayoría las jóvenes acceden a la educación supe- 
rior, y se encuentran en condiciones de ingresar al mundo laboral en posiciones más ventajosas. Como hemos observado, en sus trayectorias biográficas postergan la conyugalidad y la fertilidad hasta la adultez, aunque no necesariamente la entrada en la sexualidad activa. Sus trayectorias reproductivas se encuentran poco interferidas por la existencia del ENP, y preventivamente más protegidas. Sugerimos que esto puede ser interpretado como una perspectiva más individualizada en la configuración de ciertas trayectorias femeninas en la sociedad chilena.

En tanto, las jóvenes en los grupos socioeconómicos de más bajos ingresos pueden cruzar barreras educacionales intermedias, y muy difícilmente la que les separa de la educación superior, y no se encuentran en condiciones de ingresar al mundo laboral, sino precariamente. En sus trayectorias biográficas tienden a postergan menos la conyugalidad, aunque sí procuran hacerlo con su fertilidad -como se colige del hecho que una proporción importante declara haber experimentado un ENP. En esta perspectiva, sus trayectorias reproductivas se encontrarían interferidas por la existencia del ENP, y es probable que comiencen sus prácticas preventivas justamente después de esta experiencia.

\section{Referencias}

1. Ministerio de Salud. (2006) Informe de resultados II Encuesta Nacional de Calidad de Vida y Salud. Chile, 2006. http://epi.minsal.cl/epi/html/sdesalud/calidaddevida2006/Informe\%20Final\%20Encuesta $\% 20$ de $\% 20$ Calidad\%20de\%20Vida\%20y\%20Salud\%202006.pdf

2. Ministerio de Salud. Informe de Encuesta Nacional de
Salud. Chile 2009-2010.http://www.redsalud.gov.cl/ portal/docs/page/minsalcl/g_home/submenu_portada_2011/ens2010.pdf

3. Junta Nacional d Jardines Infantiles, UNICEF y UNESCO. (2010) Informe final Encuesta Nacional de Primera Infancia, ENPI 2010. Santiago, Chile.

4. Bajos N, Moreau C, Leridon H, Ferrand M. Pourquoi le nombre d'avortements n'a-t-il pas baissé en France depuis 30 ans? Population et Sociétés 2004; 407: 1-4.

5. Palma I. "Trayectorias sexuales, preventivas y sociales en el embarazo no previsto de los segmentos juveniles en Chile." Última década 2010; vol.18, n 33: 87-113.

6. Bourdieu P. (1990). El sentido práctico. Madrid, Taurus.

7. Johnson AM, Wadsworth J, Wellings K, Field J. Sexual Attitudes and Lifestyles. (1994). Oxford: Blackwell Scientific Press.

8. Espinoza O, González L, Uribe D. Movilidad Social en Chile: El Caso del Gran Santiago Urbano. Revista de Ciencias Sociales 2009; XV (4): 58-606.

9. Torche F. Unequal but Fluid: Social Mobility in Chile in Comparative Perspective. American Sociological Review 2005; 70 (3): 422-50.

10. Bozon M, Kontula O. Initiation sexuelle et genre: comparaison des évolutions de douze pays européens. Population 1997; 52 (6): 1367-400.

11. Heilborn ML, Aquino E, Bozon M, Riva Knauth D. O aprendizado da sexualidade. Reproduçâo e trajetorias socials de jovens brasileiros (2006). Editora Fiocruz; Editora Garamond. Rio de Janeiro, Brasil.

12. Robles F. El Desaliento Inesperado de la Modernidad. Molestias, Irritaciones y Frutos Amargos de la Sociedad del Riesgo (2000). Universidad de Concepción. Ed. Sociedad Hoy. Concepción, Chile.

13. Programa de Naciones Unidas para el Desarrollo (2002). Desarrollo humano en Chile, Nosotros los chilenos, un desafío cultural. PNUD. Santiago, Chile. 